\title{
Monte-Carlo imaging for optical interferometry
}

Ireland, Michael, Monnier, John, Thureau, Nathalie

Michael J. Ireland, John D. Monnier, Nathalie Thureau, "Monte-Carlo imaging for optical interferometry," Proc. SPIE 6268, Advances in Stellar Interferometry, 62681T (28 June 2006); doi: 10.1117/12.670940

Event: SPIE Astronomical Telescopes + Instrumentation, 2006, Orlando, Florida, United States 


\title{
Monte-Carlo Imaging for Optical Interferometry
}

\author{
Michael J. Ireland ${ }^{a}$, John D. Monnier ${ }^{b}$ and Nathalie Thureau ${ }^{b}$ \\ ${ }^{a}$ Caltech, MC 150-21,1200 E. California Blvd., Pasadena, CA 91125, USA; \\ ${ }^{b}$ Department of Astronomy, University of Michigan, 501 East University Av., Ann Arbor, \\ MI 48109, USA
}

\begin{abstract}
We present a flexible code created for imaging from the bispectrum and $V^{2}$. By using a simulated annealing method, we limit the probability of converging to local chi-squared minima as can occur when traditional imaging methods are used on data sets with limited phase information. We present the results of our code used on a simulated data set utilizing a number of regularization schemes including maximum entropy. Using the statistical properties from Monte-Carlo Markov chains of images, we show how this code can place statistical limits on image features such as unseen binary companions.
\end{abstract}

Keywords: astronomical software,aperture synthesis imaging, optical interferometry, Bayesian statistics

\section{INTRODUCTION}

It is well known that a large class of images can be consistent with a particular interferometric data set. This is more true for optical inferferometry than radio interferometry, due to the general unavailability of absolute visibility phase. An imaging algorithm such as CLEAN or Maximum Entropy combined with self-calibration attempts to find the 'best' possible image consistent with the interferometric data. Both finding this 'best' image and interpretation of features within the image can be difficult, and in general requires some kind of regularization. Regularization punishes images that look 'bad' (such as having too much unresolved structure) to find a compromise between lowering the $\chi^{2}$ statistic and achieving an optimal regularization statistic.

The imaging code MACIM described in this paper is a Monte-Carlo Markov chain algorithm that aims to both reliably find the global minimum of a regularized $\chi^{2}$ statistic in image space, and to characterize this minimum. The algorithm can operate without any regularization to find images that are optimal in the Bayesian sense. In this mode, the code can also characterize the joint probability density of images consistent with the data. Alternatively, the code can combine model-fitting and imaging or use novel regularizations based on $a$ priori imaging constraints such as the expected existence of connected regions of zero flux.

\subsection{Markov Chains and Bayesian Inference}

Bayes theorem states that the probability that a model $\theta$ (i.e. an image in our context) is correct given a given data set $\mathrm{D}$ (which includes errors on the data) is: ${ }^{1}$

$$
p(\theta \mid D)=\frac{f(D \mid \theta) p(\theta)}{f(D)}
$$

where

$$
f(D)=\int f(D \mid \theta) p(\theta) d \theta .
$$

Here $p(\theta)$ is the prior distribution of $\theta$, and $p(\theta \mid D)$ is the posterior distribution. In the case of independent Gaussian errors, the likelihood function $f(D \mid \theta)$ takes a multivariate Gaussian form:

Further author information: E-mail: mireland@gps.caltech.edu 


$$
f(D \mid \theta) \propto \exp \left(\sum\left(D_{m}(\theta)-D\right) / 2 \sigma_{i}^{2}\right)=\exp \left(\chi^{2} / 2\right)
$$

Here $D_{m}(\theta)$ is the model data $\left(V^{2}\right.$, bispectrum) corresponding to the image $\theta$. For the context of imaging, a regularization technique is contained in the pre-determined prior distribution $p(\theta)$.

When imaging or tackling many other problems with high dimensionality, the integral in Equation 2 can not be evaluated in a reasonable time, so it is not possible to explicitly evaluate Equation 1. An alternative to explicit evaluation is to use a Monte-Carlo Markov Chain technique to sample the regions of image space where $p(\theta \mid D)$ is highest. ${ }^{1}$ The distribution of images in the resultant Markov Chain $\theta_{j}$ then becomes a discrete version of the posterior distribution $p(\theta \mid D)$ from which inferences on the set of possible images can be made.

\section{MACIM IMAGING ALGORITHM}

\subsection{General Algorithm}

The general algorithm used by MACIM is a simulated annealing algorithm with the Metropolis sampler. ${ }^{1,2}$ The $^{2}$ image state space $\theta_{j}$ at iteration $j$ consists of the set of pixel vectors $\left\{p_{i}\right\}_{j}$ for all flux elements $i$ with $1 \leq i \leq \lambda$. $\lambda$ is the total number of flux elements. The flux in the image is constrained to be equal to 1 , unless model fitting of Section 2.3 is used. The vectors $p_{i}$ exist on a finite square grid with resolution at least $\lambda / 4 \max (\{B\})$, with $\max (\{B\})$ the maximum baseline length. There are two classes of steps that the algorithm can take. The first class of step moves a flux element, i.e. it randomly chooses a flux element $I$, modifies $p_{I}$ to form the tentative state $\left\{q_{i}\right\}=\left\{p_{1}, p_{2}, \ldots, p_{I}+s, \ldots, p_{n}\right\}$ for some step $s$, chosen to be in a random direction. Given a temperature $T$, the modification to the image state is accepted with a probability:

$$
p(j, j+1)=\min \left(1, \exp \left(\frac{\chi^{2}\left(\left\{p_{i}\right\}_{j}\right)-\chi^{2}\left(\left\{q_{i}\right\}\right)}{2 T}+\alpha \Delta R\right)\right)
$$

Here the $\chi^{2}$ function is the total $\chi^{2}$ function calculated directly from the interferometric data in oifits format ( $V^{2}$, bispectrum, complex visibility). $\Delta R$ is the change in the regularization parameter $R$ and $\alpha$ is a regularization scaling parameter. If the tentative state is accepted, then $\left\{p_{i}\right\}_{j+1}$ is set to $\left\{q_{i}\right\}$. Otherwise, we set $\left\{p_{i}\right\}_{j+1}=\left\{p_{i}\right\}_{j}$.

The tentative moves for $p_{i}$ include several different types of flux steps $s$ : moving one or several pixels along one of the image axes, moving the flux unit anywhere in the image, or moving to the location of another randomly selected flux element. Large steps in general have a smaller probability of success than small steps. For this reason, the step type for the tentative move is chosen so that on average the probability of accepting the tentative state is between 0.2 and 0.45 . For all steps $s$, the probability of choosing the tentative reverse transition at random is equal to the probability of choosing the forward transition.

The configuration space entropy (i.e. the logarithm of the image degeneracy) does not explicitly come into Equation 4, but does enter the picture if one wishes to find the most probable, or mode image. To understand why this is, consider the image representation $\left\{N_{k}\right\}$ where $N_{k}$ represents the number of flux elements in pixel $k$. Two images with the same $\left\{N_{k}\right\}$ are equal but can be degenerate, as each can be formed by a number of possible state vectors $\left\{p_{i}\right\}$. We will follow the notation of Ref. 3 , and call this the multiplicity $W$ :

$$
W=\frac{\lambda !}{N_{1} ! N_{2} ! \ldots N_{n} !} .
$$

Here $n$ is the total number of pixels in the image. Changing the total number of image elements $\lambda$ was done in Ref. 3 by assuming a uniform prior on $\lambda$ : all numbers of non-zero flux elements were assumed equally probable. This means that the normalized prior distribution of $\left\{N_{k}\right\}$ is given by:

$$
p\left(\left\{N_{k}\right\}\right)=\frac{W}{n^{(1-\delta) \lambda}},
$$


with the parameter $\delta=0$. In general, this prior distribution for $\left\{N_{k}\right\}$ with $\delta=0$ does not give 'sensible' images when $\lambda$ was permitted to vary (the Markov chain would converge at very high $\chi^{2}$ and a low number of elements). Therefore, non-zero values of, $\delta$ can be input as an optional parameter. Note that $\delta=1$ is equivalent to the prior distribution for $p\left(\left\{N_{k}\right\}\right)$ that all configurations are equally probable, as opposed to all values of $\lambda$ being equally probable.

The second class of step consists of adding a new flux element in pixel $K$ or removing flux element $I$ (i.e. changing $\lambda$ ). This step is intrinsically asymmetrical, as the probability of the reverse step not equal to the forward step. However, for $\delta=0$, the ratio of the probabilities of the forward and reverse steps is equal to the inverse of the ratio of the prior probability from Equation 6, meaning that Equation 4 is still appropriate for determining the Metropolis algorithm acceptance probability. For other values of $\delta$, the exponential function in Equation 4 is multiplied by $n^{\delta}$ when adding a flux element, and divided by $n^{\delta}$ when removing a flux element. For removing flux element $I$, we use $\left\{q_{i}\right\}=\left\{p_{1}, p_{2}, \ldots, p_{I-1}, p_{I+1}, \ldots, p_{n}\right\}$, and for adding to pixel $K$ we use $\left\{q_{i}\right\}=\left\{p_{i}, K\right\}$.

The annealing temperature $T$ is modified based on the reduced $\chi^{2}, \chi_{r}^{2}$, according to the following algorithm:

$$
T_{j+1}=T_{j}+\frac{\left(\chi_{r, j}^{2}-\gamma T_{j}\right)\left(1-\chi_{t}^{2} / \chi_{r, j}^{2}\right)}{\Delta j}
$$

The parameter $\gamma$ is always greater than 1 (set to 4 by default). The other parameters are the reduced $\chi^{2}$ target $\chi_{t}^{2}$ and the timescale of temperature changes $\Delta j$. This algorithm fixes $T_{j}$ to be near $\chi_{r, j}^{2} / \gamma$ during convergence, and then fixes $\chi_{r}^{2}$ to be near $\chi_{t}^{2}$ once the algorithm has converged. A minimum temperature limit $T_{\min }$ can also be placed on $T_{j}$.

\subsection{Regularizers}

There are two currently implemented regularizers in MACIM, although many are possible given that no derivative is required, as is often the case for other imaging algorithms. The first regularizer is simply the maximum entropy regularizer $R=\log (W)$. With a sufficient number of flux elements, MACIM can therefore be used to find the maximum entropy regularized image. The second implemented regularizer is a dark interaction energy regularizer. This regularizer is the sum of all pixel boundaries with zero flux on either side of the pixel boundary. Inspired by the Ising model, this regularizer encourages large regions of dark space in-between regions of flux and represents a means to utilize a priori knowledge of source structure.

\subsection{Model Fitting}

For certain astrophysical targets, a combination of model-fitting and imaging can significantly aid in data interpretation. An example of this is the point-source plus extended flux images of VY Cma and NML Cyg in Ref. 4 where the use of a maximum entropy prior with a central point source changed the image morphology significantly. Model fitting is combined with imaging in MACIM by varying model parameters simultaneously with flux movement. Currently, the only implemented model fitting option is a centrally-located uniform disk (or point source) that takes up some fraction of the total image flux, and an over-resolved (background) flux component. This model has three parameters: flux fraction of the central source, diameter of the central source and over-resolved flux fraction. Any of these parameters can be fixed or be allowed to move freely according to the Metropolis-Hastings algorithm. The parameter step sizes are chosen so that the probability of accepting the tentative new parameter is on average 0.3 .

\subsection{Specific Modes of Operation}

There are several ways in which MACIM can create images:

- Bayesian mean map. This is the default mode of operation, with the settings $T_{\min }=1, \chi_{t}^{2}=0, \lambda$ fixed at the number of input degrees of freedom and no regularization. Starting from an initial map (by default a point source), the simulated annealing algorithm converges to a global minimum where as long as $\chi_{r}^{2}<\gamma$ (default $\gamma=4$ ) we have $T=1$. Once we have $T=1$, the properties of Markov chains enable the full posterior distribution of images to be sampled. Optionally, the full chain can be output instead of just the Bayesian mean. 
- Variable- $\lambda$ Bayesian mean map. By choosing $\lambda_{\min }<\lambda_{\max }$, the number of image elements is permitted to vary. Due to frequent convergence problems, $\delta$ is set to 0.1 rather than 0 by default.

- Bayesian mode map. This output is also output whenever the Bayesian mean map is output. The average of a number of images near the maximum of $p\left(\left\{N_{k}\right\}\right)$ can be output, giving a variant of a maximum-entropy map (a maximum multiplicity map). Due to the quantization of flux, averaging a number of images (say, $10 \%$ of the final chain) about the mode is more aesthetically pleasing than the single mode map. An alternative to this kind of averaging is using the single mode map to adaptively bin the image plane according to the level of quantization noise.

- Pseudo-maximum entropy map. By setting $T_{\min }=0, \chi_{t}^{2}=1$ and fixing $\lambda$ to a large number (e.g. double the number of input degrees of freedom), multiplicity (which converges to entropy for large $\lambda$ ) is maximized while fixing $\chi_{r}^{2}=1$. Three kinds of potentially useful maps are simultaneously output in this case: the mean map, the mode map and the 'maximum entropy' map, which contains the same number of images as the mode map but weights the multiplicity so that the mean $\chi_{r}^{2}$ in the final image is 1.

- Regularized map. In general, model fitting and dark interaction energy regularization is most easily performed using a fixed $\lambda$. Clearly a large range of possible input parameters are possible here, depending on the exact nature of any a priori information.

\subsection{Difficulties and Future Work}

The seemingly greatest difficulty in using MACIM to make images is choosing the value of $\lambda$ (or $\delta$ if $\lambda$ is allowed to vary). One argument for an optimal $\lambda$ choice comes from the requirement that MACIM converges and well-samples the posterior distribution.

The optimal value of the acceptance probability $p(j, j+1)$ is thought to be in the range of 0.2 to $0.5 .^{1}$ For acceptance probabilities outside this range, the Markov Chain samples the posterior distribution at a much slower rate. Acceptance probabilities in this range can only be found at moderate values of $\lambda$. For this reason, MACIM can not well sample the posterior distribution in the high- $\lambda$ limit (where it becomes just like for the MEM algorithm) or in the low- $\lambda$ limit (as occurs if $\delta$ is set near zero).

Another argument for optimal $\lambda$ may be the desire that the mean value of $\chi_{r}^{2}$ is 1.0. In principle, there is a minimum in mean $\chi_{r}^{2}$ (generally less than 1.0) at some value of $\lambda=\lambda_{\min }$, and $\chi_{r}^{2}$ increases on either side of this minimum. ${ }^{3}$ Therefore, there should be two $\lambda$ values for which $\chi_{r}^{2}=1$. However, the lower- $\lambda$ value for mean $\chi_{r}^{2}=1$ can not be well sampled, because of the very high barriers to flux movement or adding/removing flux elements. Certainly this problem will require more work for either completely automatic operation of MACIM or at least a well-defined knowledge of the influence of $\lambda$ on deriving statistical inferences from the output MACIM Markov Chain.

\section{SOFTWARE IMPLEMENTATION}

MACIM is written in the c programming language, with an option for multi-threaded operation (multiple Markov chains running simultaneously, which are combined on completion). The transform between image-space and complex visibility is stored in memory as vectors containing $\exp \left(i u_{m} x_{k}\right)$ and $\exp \left(i v_{m} y_{k}\right)$ for baselines $m$ and pixels $k . u_{m}$ and $v_{m}$ are the standard $u$ and $v$ coordinates for baseline $m$. Splitting the pixel coordinates into $x_{k}$ and $y_{k}$ in this fashion means that only $2 M_{b} \sqrt{n}$ complex numbers need to be stored in memory (with $M_{b}$ the number of baselines). At each iteration, the mathematical functions required are limited to elementary arithmetic operations and one evaluation of the exponential function (Equation 4). No evaluations of FFTs, trigonometric functions or square roots are required. For this reason, the millions of iterations required to characterize the posterior distribution can be run on a $2 \mathrm{GHz}$ class computer in several minutes for a typical modern interferometric data set.

Only one argument is required to run MACIM: the input oifits file name. However, the default image size of $\lambda / \min (B)$ with $\min (B)$ the minimum baseline length is often not appropriate for a given data set. The maximum number of image elements $\lambda_{\max }$ and the pixel scale are other parameters that sometimes should be tweaked for 


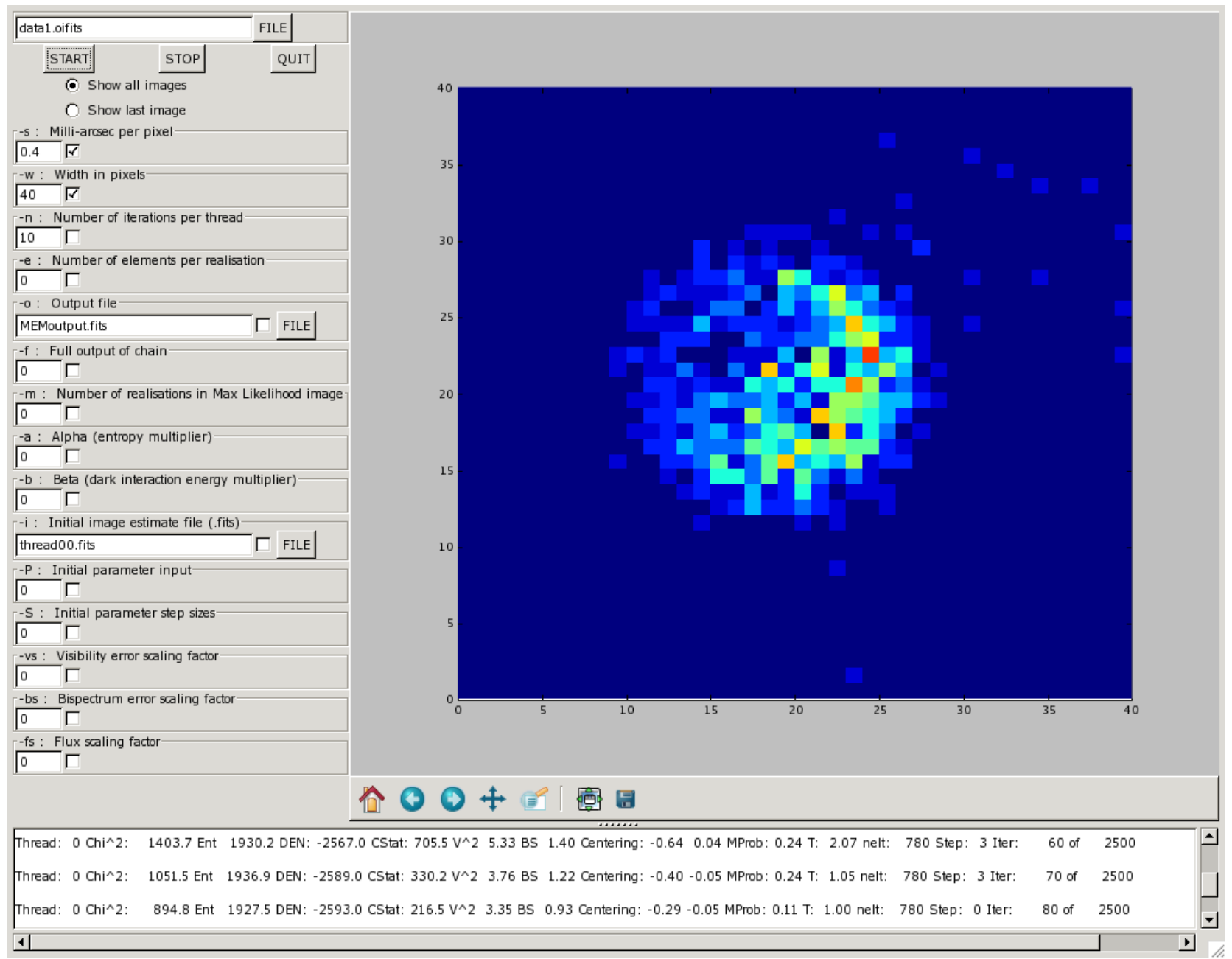

Figure 1. A screen shot of the MACIM GUI, showing a single image from the Markov Chain at a point where the algorithm has almost converged. The parameters in the lower window include the algorithm temperature $T$, the $\chi_{r}^{2}$ values for $V^{2}$ (V)̂े) and bispectrum (BS).

optimal performance. Furthermore, if convergence is too time consuming, then it can be advantageous to run MACIM with a fixed small $\lambda$ until convergence, and then increase $\lambda_{\max }$ with the converged image as an initial model fits file.

MACIM is run via the unix command line or a python-based graphical user interface (GUI). A screen-shot of the GUI is shown in Figure 1, demonstrating the display of an image $\left\{N_{k}\right\}_{j}$, the output from MACIM and text boxes for easy optional parameter input. There are also IDL tools available in the MACIM distribution for graphically displaying the images $\left\{N_{k}\right\}_{j}$ and the $\chi_{r}^{2}$ values as MACIM converges and explores the global minimum. MACIM has been successfully tested on solaris, linux and Mac OS X platforms.

\section{EXAMPLES OF ALGORITHM PERFORMANCE}

MACIM has been tested on previous beauty contest data sets, data sets from several aperture masking experiments and data from the IOTA interferometer. In this section, we will examine the algorithm's performance on an Imaging Beauty Contest data set from 2004

The first 2004 data set was chosen because the signal-to-noise for the second data set was so high that it was hard to tell apart any two images that fit the data adequately. Figure 2 compares the model image and the 

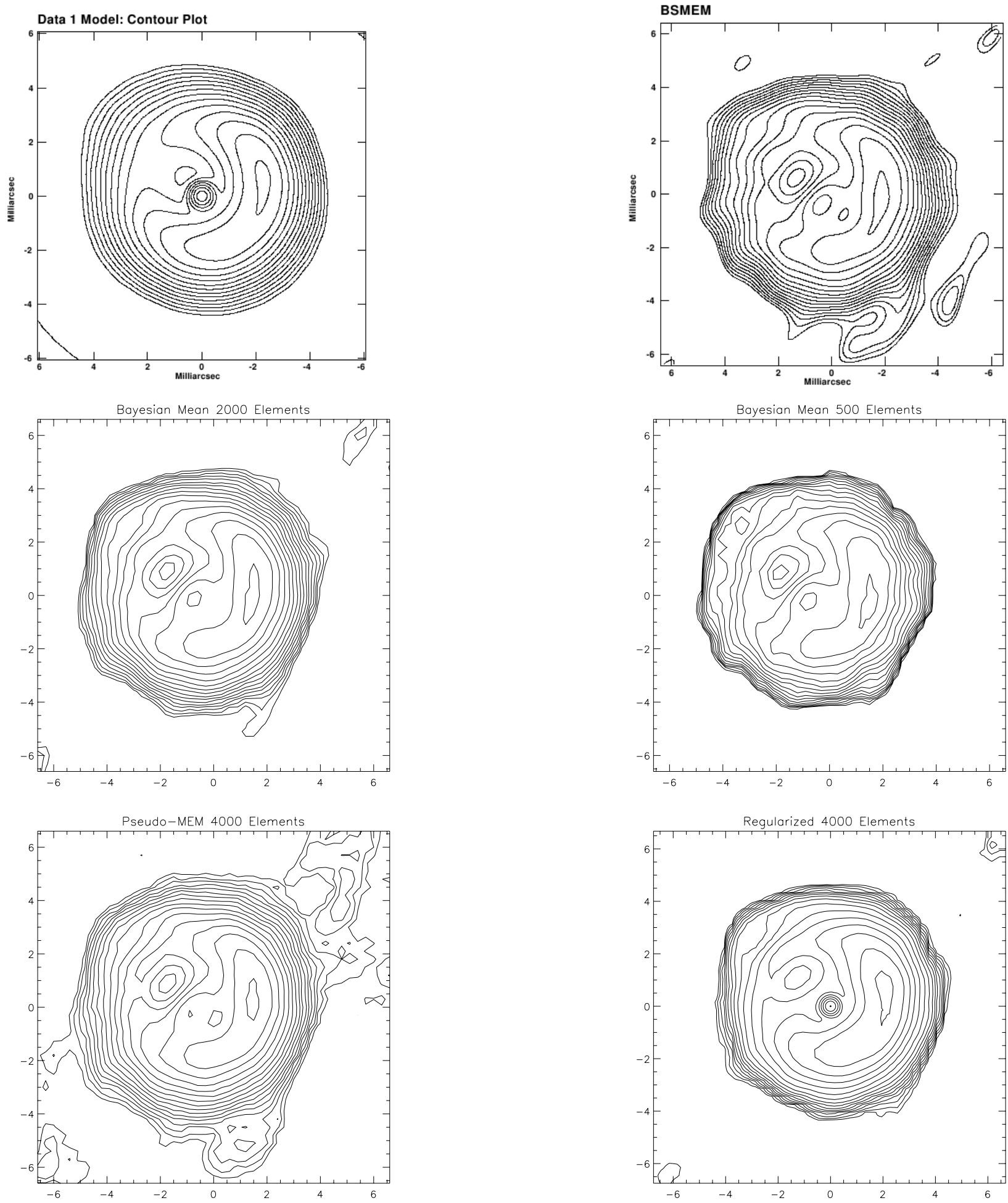

Figure 2. Top row: Model data set 1 from Ref. 5, with the reconstructed image from the 'winning' algorithm BSMEM. Middle row: Mean images constructed with 2000 and 500 flux elements. The mean $\chi_{r}^{2}$ of the 9000 images that make up these means are 1.0 and 0.87 respectively. Bottom row: A 'pseudo-MEM' map formed by using many (4000) elements, fixing $\chi_{r}^{2}=1$, and allowing the total flux to be 1.03 rather than 1.0, and a regularized map that includes knowledge of a central point-source (see text). Contours represent factors of $\sqrt{2}$ in surface brightness. 
output from the 'winning' algorithm BSMEM to four output images from MACIM.

The 'pseudo-MEM' MAP is very similar to the BSMEM map. It was created by choosing the optimal value of $\alpha$ for the MEM regularizer so that the mode image had a $\chi_{r}^{2}$ of 1 . This 'mode' image contained a mean of 900 images (10\% of those saved in memory) to reduce background noise and is a default output. Note, however, that in order to reproduce the relatively high background levels in BSMEM, the total image flux had to be set to 1.03. This is a weakness of standard MEM algorithms that find their roots in radio interferometry, where the zero-baseline correlation (i.e. the total flux) is assumed unknown.

The Bayesian mean maps with $\lambda=2000$ and $\lambda=500$ are both very similar to the MEM map. However, there is essentially zero background in the map made with $\lambda=500$, because of the large $\chi^{2}$ penalty of $1 / 500$ th of the flux moving out of the central region, compared to $1 / 2000$ th of the flux moving out of this region. Limiting the number of flux elements is therefore a powerful regularizer. For this data set, one could argue that the optimal number of image elements is about 2000, because with 2000 elements the mean value of $\chi_{r}^{2}$ is 1.0 at unity temperature.

Using the full output of the chain with $\lambda=2000$, we can answer the question "Is the feature in the top right hand corner real?" with some degree of statistical robustness. Of course, the answer will be slightly different depending on the value of $\lambda$, so answering these kinds of questions with MACIM is still work-in-progress. Given that there are 2000 flux elements, an appropriate question phrasing is "What is the confidence level for the top right feature containing more than 1/2000th of the flux". By adding up the flux elements in a $3 \times 3$ pixel region for each step in the Markov Chain and calculating the fraction of time there is non-zero flux, the confidence level for the feature is only $54 \%$.

The possibility of strong regularization with MACIM is also demonstrated in Figure 2. The regularized image is a mean map that includes the dark interaction energy regularizer. The chain began with the converged output of a previous chain and a point source with fractional flux 3\% (which could be inferred from e.g. spectral energy distribution fitting). The point source flux was left free in the Markov-Chain process, and reached an equilibrium flux value of $2.6 \pm 0.2 \%$. The dark interaction energy (although maybe not applicable to this image, which has a significant background) gives the image a sharp edge. The difference between a point-source model and an image where many flux elements are in the same pixel is caused by the multiplicity $W$. There is a very small chance that many (in this case 100 ) flux elements can congregate in a single pixel, so the presence of a point-source becomes strong a priori knowledge that influences the final image.

\section{CONCLUSION}

We have demonstrated that the Markov Chain imager MACIM can reproduce images from oifits data with at least equivalent fidelity to competing algorithms. The main benefit of MACIM are the simulated annealing algorithm that can converge where self-calibration does not, and the flexibility in regularization techniques. The source code, GUI, IDL utilities and compilation instructions for MACIM are freely available at http://www.gps. caltech.edu/ mireland/MACIM.

\section{ACKNOWLEDGMENTS}

M.I. would like to acknowledge Michelson Fellowship support from the Michelson Science Center and the NASA Navigator Program.

\section{REFERENCES}

1. D. Gamerman, Markov Chain Monte Carlo: Stochastic Simulation for Bayesian Inference, pp. 39,165. Chapman \& Hall/CRC, 1997.

2. P. Brémaud, Markov Chains: Gibbs Fields, Monte Carlo Simulation, and Queues, pp. 290-332. Springer Science+Buisiness Media, Inc., 1999.

3. E. C. Sutton and B. D. Wandelt, "Optimal Image Reconstruction in Radio Interferometry," ApJS 162, pp. 401-416, Feb. 2006. 
4. J. D. Monnier, R. Millan-Gabet, P. G. Tuthill, W. A. Traub, N. P. Carleton, V. Coudé du Foresto, W. C. Danchi, M. G. Lacasse, S. Morel, G. Perrin, I. L. Porro, F. P. Schloerb, and C. H. Townes, "High-Resolution Imaging of Dust Shells by Using Keck Aperture Masking and the IOTA Interferometer," ApJ 605, pp. 436461, Apr. 2004.

5. P. R. Lawson, W. D. Cotton, C. A. Hummel, J. D. Monnier, M. Zhao, J. S. Young, H. Thorsteinsson, S. C. Meimon, L. M. Mugnier, G. Le Besnerais, E. M. Thiebaut, and P. G. Tuthill, "An interferometry imaging beauty contest," in New Frontiers in Stellar Interferometry, Proceedings of SPIE Volume 5491. Edited by Wesley A. Traub. Bellingham, WA: The International Society for Optical Engineering, 2004., p.886, W. A. Traub, ed., p. 886, Oct. 2004. 